\title{
Facial-Based Cardiovascular Diseases Identification for Periodontal Disease Patients using Deep Learning Modified Neural Networks
}

\author{
K .G. Rani Roopha Devi ${ }^{\mathrm{a}}$, Dr.R. Murugesan ${ }^{\mathrm{b}}$, and Dr.R.MahendraChozhan ${ }^{\mathrm{c}}$

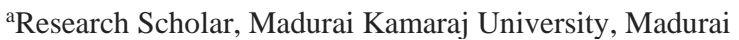 \\ ${ }^{\mathrm{b}}$ Associate Professor, Department of Computer Science, C.P.A College, Bodinayakanur \\ 'Doctor, Chozhan Dental Clinic, Periyakulam, Kodaikanal, Lakshmipuram.
}

Article History: Received: 11 January 2021; Accepted: 27 February 2021; Published online: 5 April 2021

\begin{abstract}
Challenging factor involved in the prediction of Cardio vascular Disease(CVD)plays a major role in a human health organization. Many prediction strategies still problematic for high-risk patients, most commonly the patient with continuous monitoring like elderly care and other correlated decease can lead to sudden Cardiovascular death (stroke, periodontal disease, etc) can involve in the high-risk category. The measurement and prediction always based on various testing factors involved in blood pressure, ECG, etc. Face emotional expression is a sort of discomfort behavior that gives the impression to be a universal factor of the pain experience. The remote heart rate measurement plays an important role in high-risk patients.In this paper, the facial-based Cardiovascular diseases are identified for periodontal disease patients using deep learning modified neural networks. The patient with periodontal disease can possible increased chance of Cardiovascular diseases. the classification can also be based on other testing consequences.
\end{abstract}

Keywords: Periodontal disease, Artificial Bee Colony,Particle Swarm Optimization, GFT-SDM, PCA-DCT.

\section{Introduction}

Periodontitis is a serious disease of the gums. It's brought about by microorganisms that have been permitted to gather on your teeth and gums[1]. As periodontitis advances, the bones and teeth can be harmed. Be that as it may, if periodontitis is dealt with right on time and legitimate oral cleanliness is kept up, the harm can be halted.The non- treated for periodontitis, can lead to serious illness, the jaw bone is sometimes destroyed due to periodontitis and other most dangerous illness of increased risk of heart disease, respiratory disease, and diabetes[2].

Numerous epidemiological examinations have explored the connection between periodontal and cardiovascular.The result shows that a potential connection exists among periodontal and cardiovascular. Oral medical services experts can recognize patients who are uninformed of their danger of creating genuine entanglements because of CVD and who are needing clinical mediation[3]. World health organization reported CVDs are the number 1 reason for death around the world.a larger number of individuals pass on yearly from CVDs than from some other reason. An expected 17.9 million individuals passed on from CVDs in 2016, speaking to $31 \%$ of every single worldwide demise. Of these passings, $85 \%$ are because of coronary failure and stroke. More than $75 \%$ of CVD passings occur in low-and center pay nations. Out of the 17 million unexpected losses (younger than 70) because of noncommunicable ailments in 2015, 82\% are in low-and center pay nations, and $37 \%$ are brought about by CVDs. Most cardiovascular illnesses can be forestalled by tending to conduct hazard factors, for example, tobacco use, unfortunate eating regimen and corpulence, physical inertia, and unsafe utilization of liquor utilizing populace wide[4]

Individuals with cardiovascular infection or who are at high cardiovascular danger (because of the presence of at least one danger factor, for example, hypertension, diabetes, hyperlipidemia, or effectively settled malady) need early discovery and the executives utilizing directing and drugs, as fitting[5].The early discovery includes the video-based heart attack detection with the help of patient facial emotional expressions. The smart detection includes the Deep learning modified neural network for the advancement with the Artificial Bee Colony-Particle Swarm Optimization (ABC-PSO)algorithm[6].

The paper has been arranged as, section 1 describes the introduction, section 2 represents the basic method about the proposed work, section 3 describes the result and analusis of the proposed work, section 4 concluded the paper.

\section{Materials and Methods}

\subsection{ABC-PSO}

Optimization problems assume a significant part in numerous logical and designing fields. Over the most recent twenty years, a few multitude knowledge calculations, for example, ant colony optimization (ACO), particle swarm optimization (PSO), and artificial bee colony (ABC) algorithm, have been created for tackling troublesome enhancement issue. Analysts have indicated that calculations dependent on swarm-intelligent have the incredible potentia and have pulled in much consideration[7].

Since the creation of the ABC calculation, it has been utilized to illuminate both mathematical and nonnumerical enhancement issues. The presentation of $\mathrm{ABC}$ calculation has been compared and some other clever calculations, for example,Genetic Algorithm(GA), differential advancement calculation. The outcomes show that 
$\mathrm{ABC}$ calculation is in a way that is better than or if nothing else similar to different techniques[8]. As of late, for improving the exhibition of $\mathrm{ABC}$ calculation, numerous variation $\mathrm{ABC}$ calculations have been created. Moreover, considering PSO has great exploitation capacity, a couple of mixture $\mathrm{ABC}$ calculations have been introduced dependent on PSO calculation. there is as yet a deficiency in ABC with respect to its solution search condition, which is acceptable at investigation however poor at exploitation[9,10]. To beat this issue, we propose a novel counterfeit honey bee state calculation dependent on molecule swarm search component. In this calculation, for improving the combination speed, the underlying populace is produced by utilizing valid statement set hypothesis as opposed to irregular determination right off the bat. Besides, so as to improve the exploitation capacity, the utilized honey bee, spectators, and scouts use the component of PSO to look through new competitor arrangements.

\subsection{GFT-SDM}

The proposed technique uses a mix of "Good Features Track" and a "Supervised Descent Method for facial highlights point detection. Utilizing these techniques the limitation of the current frameworks utilized for HR estimating framework utilizing facial video can be settled. the cyclic and non-cycling trajectories of the tracked points and to remove the non-cyclic components the current frameworks is referenced above like unreasonable limitations of the objects development and the helping issue. The wrong results are forestalled by joining the face quality appraisal method. There is have to follow the element focuses of the face and create direction by keep the record of head movement in the video which are caused because of heartbeat. The point of direction extraction just as sign preparing is to discover the cyclic and non-cycling directions of the followed focuses and to eliminate the non-cyclic components. The proposed technique uses a mix of "Good Features Track" and a "Supervised Descent Method" for facial highlights point detection. Utilizing the above strategy the constraint of the current frameworks utilized for HR estimating framework utilizing facial video can be settled. The restriction of the current frameworks is referenced above like unreasonable limitations of the objects' development and the helping issue.

$$
f_{G F T}(\boldsymbol{\delta})=\sum_{x=p_{x}}^{p_{x}+w_{x}} \sum_{y=p_{y}}^{p_{y}+w_{y}}(I(\mathbf{x})-J(\mathbf{x}+\boldsymbol{\delta}))^{2}
$$

GFT feature has been generated using equarion 1. And the SDM has been calculated using equation 2.

$$
f_{S D M}\left(x_{0}+\Delta x\right)=\left\|g\left(d\left(x_{0}+\Delta x\right)\right)-\theta_{*}\right\|_{2}^{2}
$$

The above formula was used to find SDM feature. Where $\mathrm{x}_{0}$ is the initial configuration of the landmarks in a facial image, $\mathrm{d}(\mathrm{x})$ indexes the landmarks configuration $(\mathrm{x})$ in the image, $\mathrm{g}$ is a nonlinear feature extractor, $\theta^{*}=$ $\mathrm{g}(\mathrm{d}(\mathrm{X} *))$, and $\mathrm{X} *$ is the configuration of the true landmarks has been calculated using equation 3 and 4 .

$$
\begin{aligned}
& x_{n}=x_{n-1}+\partial_{n-1} \sigma\left(x_{n-1}\right)+\beta_{n-1} \\
& \partial_{n-1}=-2 \times \mathrm{H}^{-1}\left(x_{n-1}\right) \times \mathrm{J}^{\mathrm{T}}\left(x_{n-1}\right) \times g\left(d\left(x_{n-1}\right)\right) \\
& \beta_{n-1}=-2 \times \mathrm{H}^{-1}\left(x_{n-1}\right) \times \mathrm{J}^{\mathrm{T}}\left(x_{n-1}\right) \times g\left(d\left(x_{*}\right)\right)
\end{aligned}
$$

The wrong results are forestalled by joining the face quality evaluation procedure. There is have to follow the element focuses of the face and creative direction by keep the record of head movement in the video which are caused because of a heartbeat. The point of direction extraction just as sign preparation is to discover the cyclic and non-cycling directions of the followed focuses and to eliminate the non-cyclic segments[11,12].

\subsection{PCA-DCT}

There are head movements that are created from various sources yet just those movements brought about by blood course through the aorta mirrors the warmth rate, subsequently, the Principal Component Analysis algorithm (PCA) has been applied to the Filtered trajectories (FT) to isolate the wellsprings of head movement. PCA changes FT to another organize framework through figuring the orthogonal components PI by utilizing a load matrix LM Among these isolated parts, the most intermittent segment is the one which has a place with the heartbeat[13].

At that point Discrete Cosine Transform (DCT) is applied to all the (PI) segments to locate the most period one and select the principal consonant to get the HR. Hence the proposed strategy utilized a blend of SDM and GFT based drew closer[14].

The Proposed PCA algorithm has the following steps.

Information Normalization 
In this progression we standardize the information's in formally dressed model by utilizing Gaussian piece capacity to the highlights.

Here $\mathrm{x}$ speaks to push esteem,

y speaks to section esteem.

Ascertain Covariance

Figure Eigen esteem

Ascertain Eigen Vectors with most elevated Eigen esteem

Discover PCA esteem Highest Eigen esteem utilizing underneath recipe.

PCA $=$ eigen vectors $*$ part focus

After end of this algorithm we got one value. This heart rate value was added with age, sex, blood pressure, cholesterol and test with already trained dataset.

\subsection{DLMNN}

In this work the deep learning modified neural network has been employed, The deep learning neural network classifier has a convolution layer, pooling layer, and fully connected layer. It has weight and bias for the neural network learning structure, here the weight has been applied based on the modification of the PSO algorithm[18,19]

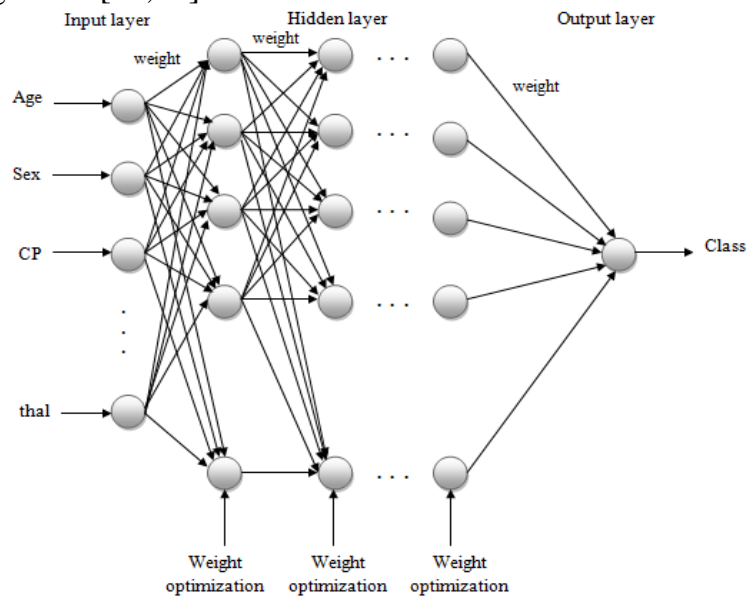

There has been plenty of work carried out to measure the heart rate based on the facial video, in 2017[14] reviewed the various research work based on heart rate estimation using video, the most of the work carried out using Photoplethysmography and Ballistocardiography, these are the method to find the heart rate by human facial video[20].

\section{Result and Analysis}

The correlation of the presentation of this approach against strategies from various performance metrics is finished. With the end goal of examination of precision estimation regarding rate can be utilized for estimation of blunder. In this work the data has been collected from the Chozhan group of dental clinics, Theni, Periyakulam, andKodaikanal The precision of a technique is higher when there is a lower mistake [15]. The perceptions show that the arrangement shows a predictable presentation in various situations. By utilizing a blend of both GFT and SDM directions, the procedure gets more directions to assess the HR design. Then again, the past strategies experience the ill effects of fewer directions and wrong directions from the information obtained in testing situations, the performance metrics of Neural Network(NN)has been obtained by the optimized Neural Network in the form of the following metrics. The figure 1 represents the number of frames conversion. 


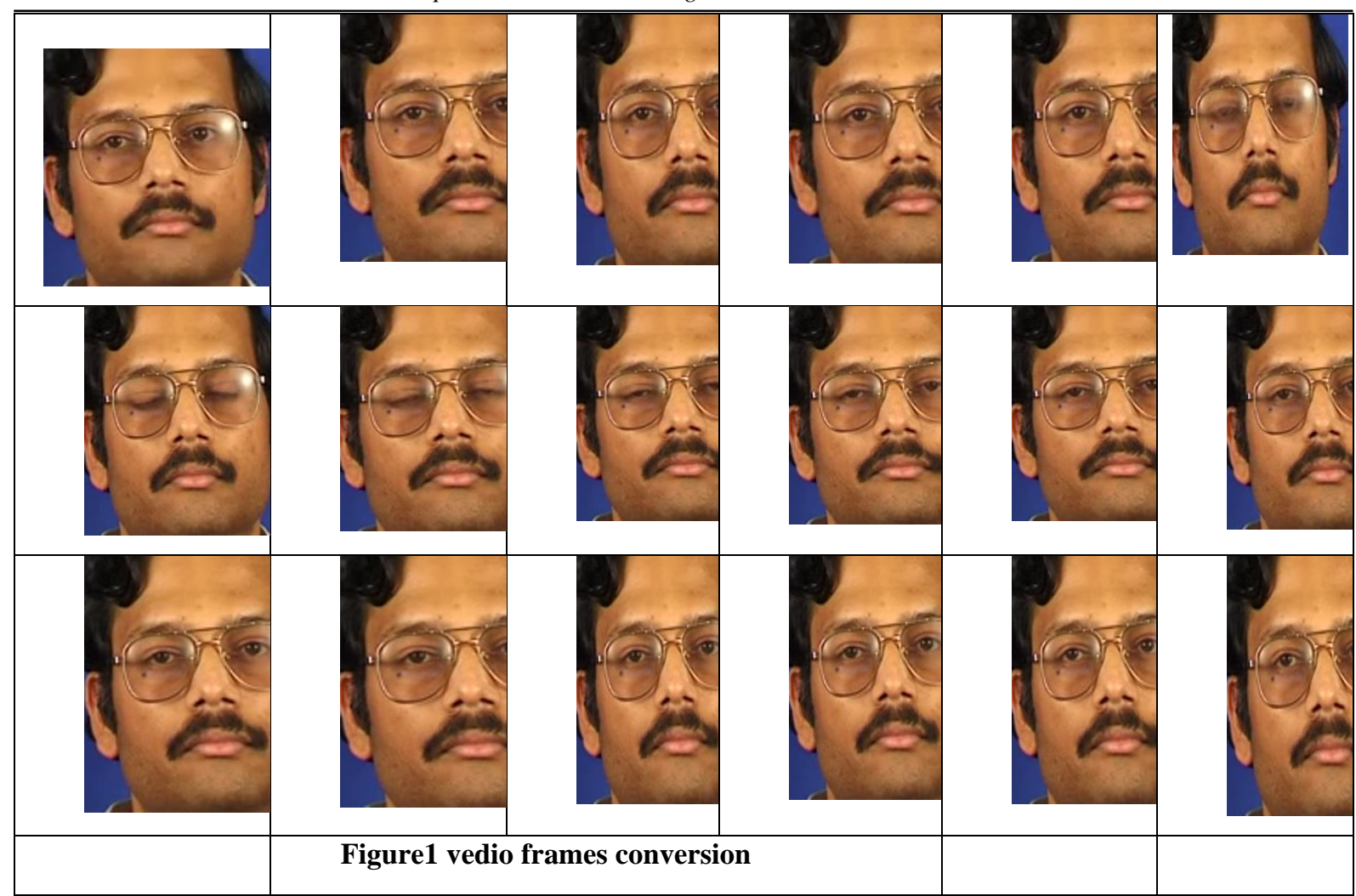

Totally 18 frames have been allowed for the second's video the figure 2 a described the Region of interest (ROI ) segmentation. $2 \mathrm{~b}$ repredents the GFT points of the ROI Voluntary head motion andthe facial expression changes in a video causes limitations in the GFT based tracking system.

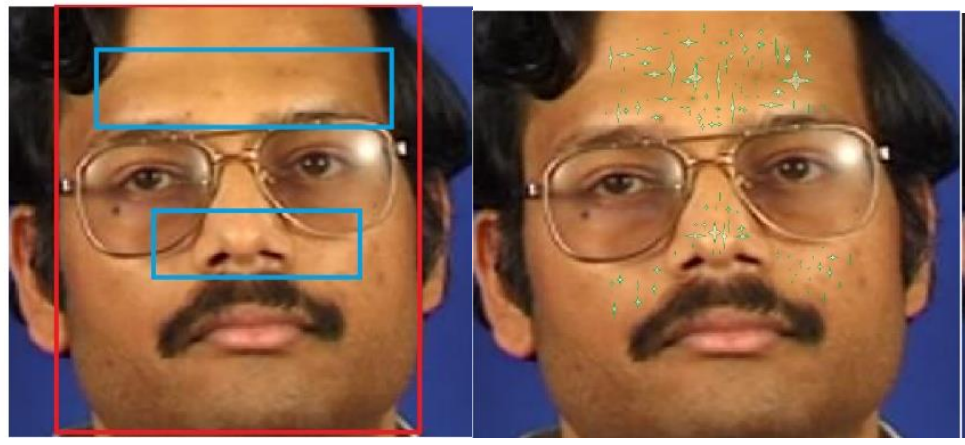

Figure 2 ( a )ROI sequence GFT-SDM

The figure 2creprents the SDM points SDM utilizes just 49 focuses when contrasted with GFT it utilizes huge no of focuses which causes to save more movement data when there is no intentional movement. Accordinglythe point is to propose a technique which joins the directions of SDM and GFT.Figure 3 represents the signal from head movements which are created from various sources yet just those movements brought about by blood flow through aorta mirrors the warmth rate, accordingly the Principal Component Analysis calculation (PCA) is applied to the sifted directions (F) to isolate the wellsprings of head motion. Among these isolated parts, the most occasional segment is the one that has a place with the

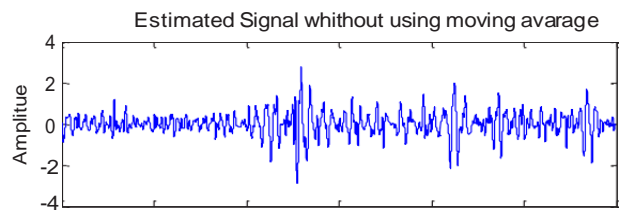

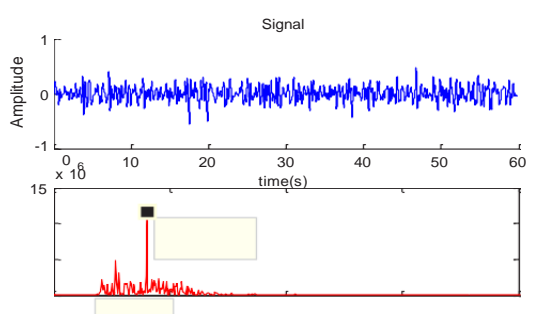

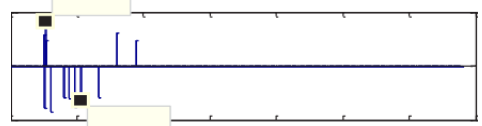


heartbeat. At that point Discrete Cosine Transform (DCT) is applied to all the (P) segments to locate the most period one. At that point utilize Fast Fourier Transform (FFT) on the converse DCT of the part and select the principal symphonious to acquire the HR. In this way the proposed strategy utilized a mix of SDM and GFT based drewnearer

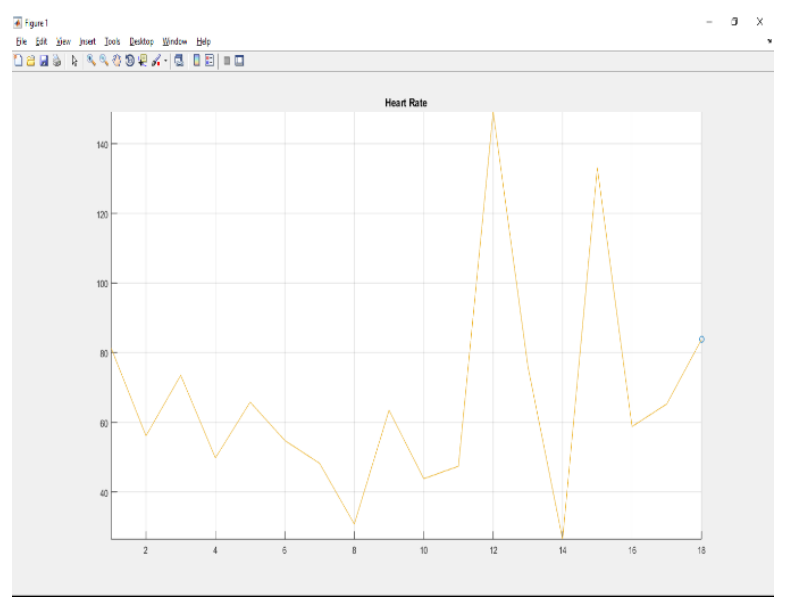

Figure 4 heart rate

The table 1 represents the performance metrics of optimized Neural Network sensitivity and specificity.

Accuracy, precision, recall, F-measure, NPV, FPR, FNR, MCC, and FRR values

Table 1 Performance metrics of Optimised NN

\begin{tabular}{|l|l|}
\hline Performance metrics & Values \\
\hline sensitivity & 0.72727 \\
\hline specificity & 0.97842 \\
\hline Accuracy & 0.96 \\
\hline precision & 0.72727 \\
\hline recall & 0.72727 \\
\hline F-measure & 0.72727 \\
\hline NPV & 0.97842 \\
\hline FPR & 0.021853 \\
\hline FNR & 0.27273 \\
\hline MCC & 0.70569 \\
\hline FRR & 0.27273 \\
\hline
\end{tabular}

\section{Conclusion}

In this paper, the employed one of the early discoveries of CVD for the patient with periodontal disease. The early discovery includes the video-based heart attack detection with the help of patient facial emotional expressions. The smart detection includes the Deep learning modified neural network for the advancement with the ABC-PSO algorithm. The framework estimates pulse from the video caught in sensible situations not at all like past existing strategies which didn't function admirably when there is willful movement of head, articulation changes, change in lightning conditions. This framework beats issue of all the above techniques by utilizing SDMbased strategy alongside GFT-base technique and gave great outcomes. The HR estimation execution of this framework is profoundly exact and solid in the difficult genuine climate as shown in the part 6 of the paper. Accordingly, this philosophy can be utilized in applications for HR estimation for constant circumstances and other delicate biometrics applications..

\section{References}

1. Lee JH, Oh JY, Choi JK, Kim YT, Park YS, Jeong SN, et al. Trends in tooth extraction incidence due to periodontal disease: results of a 12-year longitudinal cohort study in South Korea. J Periodontal Implant Sci 2017 
2. Lee JH, Lee JS, Park JY, Choi JK, Kim DW, Kim YT, et al. Association of lifestyle-related comorbidities with periodontitis: a nationwide cohort study in Korea. Medicine (Baltimore) 2015

3. Lee JH, Choi JK, Kim SH, Cho KH, Kim YT, Choi SH, et al. Association between periodontal flap surgery for periodontitis and vasculogenic erectile dysfunction in Koreans. J Periodontal Implant Sci 2017

4. Choi JK, Kim YT, Kweon HI, Park EC, Choi SH, Lee JH. Effect of periodontitis on osteoporosis development: results from a nationwide population-based cohort study (2003-2013). BMC Womens Health 2017

5. Lee JH, Kweon HH, Choi JK, Kim YT, Choi SH. Association between periodontal disease and prostate cancer: results of a 12-year longitudinal cohort study in South Korea. J Cancer 2017.

6. Sklan JE, Plassard AJ, Fabbri D, Landman BA. Toward content-based image retrieval with deep convolutional neural networks. Proc SPIE IntSoc Opt Eng 2015.

7. Rajpurkar P, Hannun AY, Haghpanahi M, Bourn C, Ng AY. Cardiologist-level arrhythmia detection with convolutional neural networks. arXiv e-print 2017:arXiv:1707.01836.

8. Litjens G, Kooi T, Bejnordi BE, Setio AA, Ciompi F, Ghafoorian M, et al. A survey on deep learning in medical image analysis. Med Image Anal 2017.

9. Kim TS, Obst C, Zehaczek S, Geenen C. Detection of bone loss with different X-ray techniques in periodontal patients. J Periodontol 2008.

10. Ronneberger, O., Fischer, P. \&Brox, T. U-net: Convolutional networks for biomedical image segmentation. In InternationalConference on Medical image computing and computer-assisted intervention, 234-241 (Springer, 2015).

11. Lee, J.-H., Hyung Kim, D., Jeong, S.-N. \& Choi, S.-H. An overview of deep learning in the field of dentistry. Diagnosis and prediction of periodontally compromised teeth using a deep learning-based convolutional neural network algorithm 48, 114-123 (2018).

12. Krois, J. et al. Deep learning for periodontal bone loss radiographic detection. Scientific reports $\mathbf{9}, 8495$ (2019).

13. Lin, T.-Y., Goyal, P., Girshick, R., He, K. \&Dollár, P. Focal loss for dense object detection. In Proceedings of the IEEE international conference on computer vision, 2980-2988 (2017).

14. Lee, J. H., Kim, D. H., Jeong, S. N. \& Choi, S. H. Diagnosis and prediction of periodontally compromised teeth using deep learning-based convolutional neural network algorithm. Journal of periodontal \& implant science 48, 114-123, 2018.

15. Lee, J. H., Kim, D. H., Jeong, S. N. \& Choi, S. H. Detection and diagnosis of dental caries using a deep learning-based convolutionalneural network algorithm. Journal of dentistry 77, 106-111, 2018.

16. Zhang, Yuzhong, Zhe Dong, Kezun Zhang, Shuangbao Shu, Fucheng Lu, and Jingjing Chen. "Illumination variation-resistant video-based heart rate monitoring using LAB color space." Optics and Lasers in Engineering 136: 106328.

17. Sharma, Prerna, Krishna Choudhary, Kshitij Gupta, Rahul Chawla, Deepak Gupta, and Arun Sharma. "Artificial plant optimization algorithm to detect heart rate \& presence of heart disease using machine learning." Artificial Intelligence in Medicine 102 (2020): 101752.

18. Ciusdel, Costin, AlexandruTurcea, Andrei Puiu, Lucian Itu, Lucian Calmac, Emma Weiss, Cornelia Margineanu et al. "Deep neural networks for ECG-free cardiac phase and end-diastolic frame detection on coronary angiographies." Computerized Medical Imaging and Graphics 84 (2020): 101749. 